\title{
Pathophysiology of Cerebral Circulatory Disorders in Idiopathic Normal Pressure Hydrocephalus
}

\author{
Totaro TAKeUChI, Hiromi GOTO*, Kenji IZAKI*, Shinya TAMURA*, \\ Masato TOMII*, Jinichi SASANUMA*, Kazushige MAENO*, \\ Yasuhiro KIKUCHI*, Jinichi KOIZUMI*, Zenichiro WATANABE*, \\ Shinichi NumaZAWA*, Yasunobu ITOH*, Kazuo WATANABE*, \\ Masanori KOJIMA**, Masayuki MishIMA**, Yuhki ONISHI**, \\ Toshimitsu OKADA**, and Takashi ARAI**
}

\author{
Department of Neurosurgery, The Gyoda General Hospital, Gyoda, Saitama; \\ Departments of *Neurosurgery and ${ }^{* *}$ Radiology, The Southern TOHOKU General Hospital, \\ Southern TOHOKU Research Institute for Neuroscience, Kohriyama, Fukushima
}

\begin{abstract}
This study was conducted to elucidate the pathologic conditions of cerebral circulatory disorders in idiopathic normal pressure hydrocephalus (iNPH). Among 44 possible iNPH patients, 40 patients underwent shunt surgery based on diagnostic flow charts plotted by the Southern Tohoku method and were evaluated to be shunt-effective at the end of the first post-surgical month. The cerebral blood flow (CBF) was measured by $\mathrm{N}$-isopropyl-( $\left.{ }^{123} \mathrm{I}\right)$-P-iodo-amphetamine single photon emission computed tomography (mean, mCBF; cortical region, CCBF; thalamus-basal ganglia region, tbCBF on autoradiography [ARG] method) and the perfusion patterns of the cerebral cortex were measured based on threedimensional stereotactic surface projection (3D-SSP) Z-score images, before and 1 month after the surgery in all 40 subjects. The $\mathrm{mCBF}$ rose significantly from $32.1 \pm 2.74 \mathrm{ml} / 100 \mathrm{~g} / \mathrm{min}$ before surgery to $39.8 \pm 3.02 \mathrm{ml} / 100 \mathrm{~g} / \mathrm{min}$ after surgery $(p<0.03)$. Investigation of the change of $\mathrm{CBF}$ revealed reductions in the CCBF ( 3 cases), tbCBF ( 9 cases), and CCBF + tbCBF ( 28 cases), with the reduced-cCBF group totaling 31 cases and the reduced-tbCBF group totaling 37 cases. Investigation of cerebral cortex hypoperfusion by 3D-SSP Z-score revealed 31 cases with hypoperfusion (frontal lobe type [19 cases], occipitotemporal lobe type [ 5 cases], mixed type [ 7 cases]) and nine cases with cortical normoperfusion (N). The pattern of reduction of the cortical blood flow on ARG method was favorably correlated with the pattern of hypoperfusion of the cerebral cortex on 3D-SSP Z-score images before surgery. A reduction of blood flow was found in the thalamus-basal ganglia region of all $\mathrm{N}$ type cases. The blood flow improved in 19 of $31(61.3 \%)$ cases of the reduced-cCBF group and in 32 of $37(86.5 \%)$ cases of the reducedtbCBF group. All of the cases without detectable improvement exhibited increased blood flow in nonreduction areas. Investigation of the hypoperfusion patterns of the cerebral cortex on 3D-SSP Z-score images, revealed a reduction or disappearance of the hypoperfusion site in 19 of $31(61.3 \%)$ cases, either no-change or a shift of the hypoperfusion site in 12 of $31(38.7 \%)$ cases, and a correlation between the pattern of cortical blood flow reduction on ARG method and the pattern of cerebral cortex hypoperfusion on 3D-SSP Z-score images after surgery. Cerebral circulatory disorders in iNPH manifest as either of two pathophysiological conditions: the "circulatory disorder of the cerebral cortical region" and the "circulatory disorder of the thalamus-basal ganglia region." Various patterns develop according to the disease stage.
\end{abstract}

Key words: idiopathic normal pressure hydrocephalus, cerebral blood flow, $\mathrm{N}$-isopropyl-(123I)-P-iodo-amphetamine, single photon emission computed tomography, three-dimensional stereotactic surface projection

Received September 26, 2006; Accepted April 18, 2007 


\section{Introduction}

Most researchers, including this author, agree that cases of idiopathic normal pressure hydrocephalus (iNPH) with decreased cerebral blood flow (CBF) before surgery undergo postoperative increases in blood flow when the shunts are effective..$^{9,12-14,17-22,24 \text {, }}$ 26-29,31,32,34) The results from reports on preoperative ischemic sites and postoperative sites of increased blood flow have varied, ${ }^{13,17,19,20,22,24,26,27,31,32)}$ however, and no firm consensus has been reached.

In this study we use N-isopropyl-(123I)-P-iodoamphetamine single photon emission computed tomography ( ${ }^{123}$ I-IMP SPECT) to compare the CBF and perfusion patterns of the cerebral cortex assessed by the three-dimensional stereotactic surface projection (3D-SSP) Z-score images at each site before and after surgery in iNPH cases effectively resolved by shunt intervention (shunt-effective cases). We also discuss the cerebral circulatory disorders induced by iNPH. The study is herein reported.

\section{Materials and Methods}

Forty of 44 patients with possible iNPH, 26 males and 14 females aged 61-81 years (mean 70.2 years), underwent lumboperitoneal (28 cases) or ventriculoperitoneal (12 cases) shunt surgery based on diagnostic flow charts of the Southern Tohoku method $^{27)}$ (Fig. 1), and were considered to be responsive at the end of the first post-surgical month. The possible iNPH patients satisfied the Japanese clinical guidelines for iNPH, including one or more of the classical triad of symptoms, ventricular dilation with Evans index $>0.3$, clear cerebrospinal fluid with normal pressure, middle age or elderly, and absence of causative disorders. ${ }^{7}$ The patients were evaluated by the revised Japanese grading scale for iNPH ${ }^{7}$ (Table 1) at the end of the first postsurgical month. Patients manifesting improvement of one point or higher in any item were regarded as responsive.

Two SPECT methods were used to measure CBF. First, CBF (mean CBF, mCBF; whole cortex, cCBF; thalamus-basal ganglia, tbCBF) was measured at each region of interest (ROI) (Fig. 2) by autoradiography (ARG) (equipment, Toshiba e-cam, Toshiba Medical Systems Co., Ohtawara, Tochigi; nuclear species, $222 \mathrm{MBq}$ intravenous injection of ${ }^{123}$ I-IMP [Perfuzamine ${ }^{\circledR}$, Nihon Medi-physics Co., Nishinomiya, Hyogo]). ${ }^{8,10)}$ Second, the perfusion patterns of the cerebral cortex were depicted by 3D-SSP Z-score images (Fig. 3) and investigated to assess the follow-
Flow-chart indication for shunting of iNPH

(Southern Tohoku method)

Possible iNPH

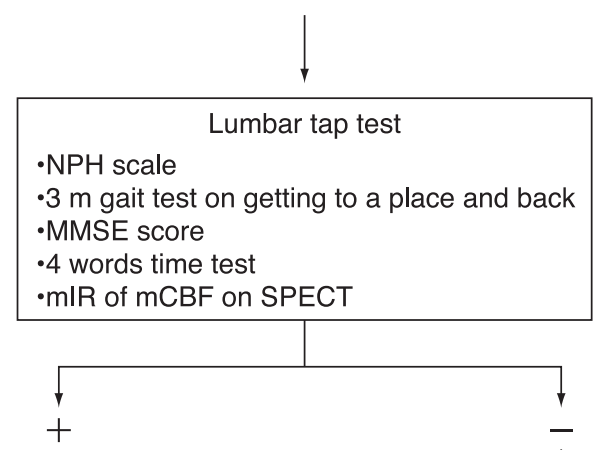

Positive if come under at least 1 item - improve more than 1 point on NPH scale - shorten more than $10 \%$ in time and step on gait test - improve more than 3 points on MMSE test - shorten more than $10 \%$ on 4 words time test . increase more than $10 \%$ in mCBF on SPECT



(LP or VP)

Fig. 1 The Southern Tohoku method flow-chart indication for shunting in patients with idiopathic normal pressure hydrocephalus (iNPH). ${ }^{30)}$ LP: lumboperitoneal, mCBF: mean cerebral blood flow, mIR: mean increasing ratio, MMSE: Mini-Mental State Examination, Ro: cerebrospinal fluid outflow resistance value, SPECT: single photon emission computed tomography, VP: ventriculoperitoneal.

ing: 1) the mCBF before and after surgery; 2) cCBF, tbCBF, and perfusion patterns of the cerebral cortex before surgery; and 3) the cCBF, tbCBF, and perfusion patterns of the cerebral cortex after surgery.

In ARG method in which supplementary regulation didn't conducted, a postoperative change of CBF of greater than $10 \%$ in the same subjects was assessed as significant because the aberration range was $\pm 6 \% .^{8,10)}$ The patterns of $\mathrm{cCBF}$ and tbCBF reduction before surgery were evaluated by comparing the reduction rates with the mean cerebral flows of normal adults at each ROI by ${ }^{123}$ I-IMP SPECT. ${ }^{30}$ ) 
Table 1 Revised Japanese grading scale for normal pressure hydrocephalus (JGSNPH-R) in the clinical guidelines for idiopathic normal pressure hydrocephalus ${ }^{7)}$

Gait disturbance $(G)$ :

normal

unstable, only subjective gait disturbance

objective gait disturbance, but independent gait

walking with canes or walker frame

walking not possible, wheelchair-bound

Dementia (D):

0 within normal range

1 only subjective attention, memory disturbance

2 objective attention, memory disturbance, but normal time, place orientation

3 objective attention, memory disturbance with time, place disorientation

4 total disorientation, conversation not possible Urinary incontinence (U):

0 absent

1 absent but pollakiuria or urinary urgency

2 sometimes, more than 1-3 times a week

3 frequent, more than 1 time a day

4 uncontrollable urinary incontinence

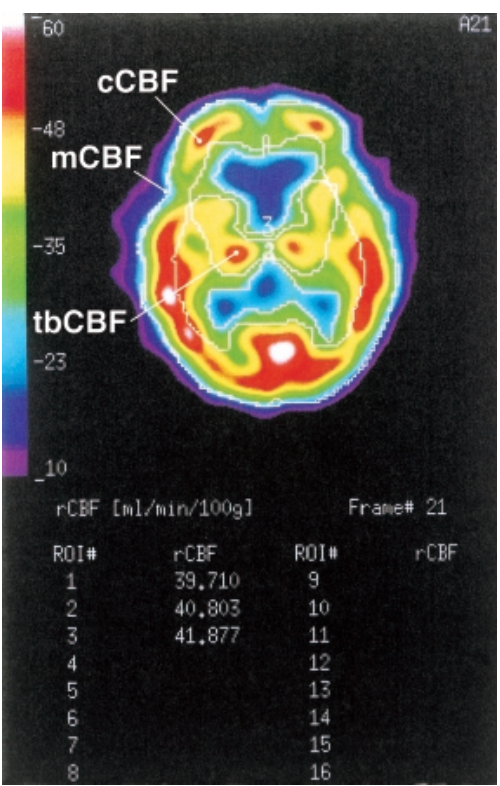

Fig. 2 Regions of interest on $\mathrm{N}$-isopropyl-( $\left.{ }^{123} \mathrm{I}\right)-\mathrm{P}$ iodo-amphetamine single photon emission computed tomography by autoradiography method. cCBF: whole cortex blood flow, mCBF: mean cerebral blood flow, tbCBF: thalamus-basal ganglia blood flow.

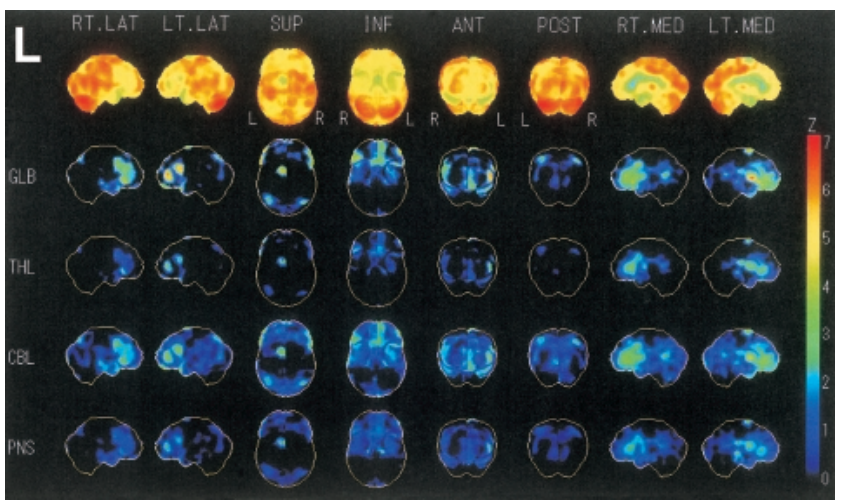

Fig. 3 Three-dimensional stereotactic surface projection Z-score images on $\mathrm{N}$-isopropyl( ${ }^{123}$ I)-P-iodo-amphetamine single photon emission computed tomography (frontal hypoperfusion type).

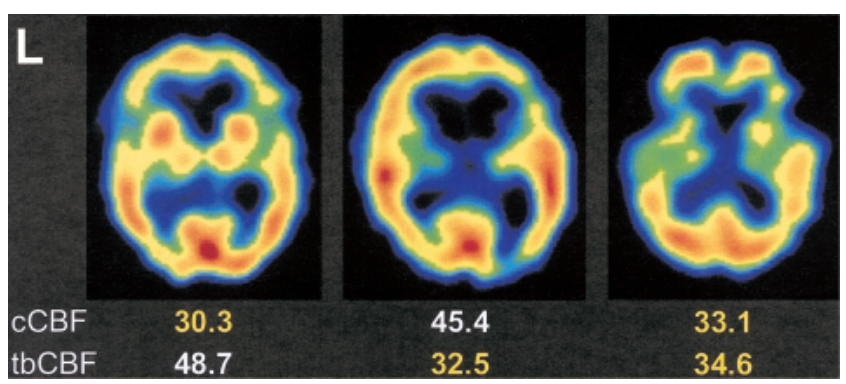

Fig. 4 Preoperative cerebral blood flow reduction patterns on N-isopropyl-( $\left.{ }^{123} \mathrm{I}\right)$-P-iodoamphetamine single photon emission computed tomography by autoradiography method. Left: type A, a 76-year-old male; center: type $B$, a 70-year-old male; right: type C, a 68-year-old female. cCBF: whole cortex blood flow in $\mathrm{ml} / 100 \mathrm{~g} / \mathrm{min}$, tbCBF: thalamus-basal ganglia blood flow in $\mathrm{ml} / 100$ g/min.

\section{Results}

\section{I. $\mathrm{mCBF}$ before and after surgery}

The mCBF rose significantly from $32.1 \pm 2.74$ $\mathrm{ml} / 100 \mathrm{~g} / \mathrm{min}$ before surgery to $39.8 \pm 3.02 \mathrm{ml} / 100$ $\mathrm{g} / \mathrm{min}$ after surgery $(\mathrm{p}<0.03)$.

II. Preoperative cCBF, tbCBF, and perfusion patterns of the cerebral cortex

The patterns of preoperative $\mathrm{CBF}$ reduction were classified into three groups: the reduced-cCBF group (type A: 3 cases), reduced-tbCBF group (type B: 9 cases), and reduced-cCBF + tbCBF group (type 
Table 2 Preoperative cerebral blood flow reduction patterns on $\mathrm{N}$-isopropyl-( $\left.{ }^{123} \mathrm{I}\right)$-P-iodoamphetamine single photon emission computed tomography by autoradiography method

\begin{tabular}{ccc}
\hline \multicolumn{1}{c}{ Type } & cCBF $(\mathrm{ml} / 100 \mathrm{~g} / \mathrm{min})$ & $\mathrm{tbCBF}(\mathrm{ml} / 100 \mathrm{~g} / \mathrm{min})$ \\
\hline $\mathrm{A}(\mathrm{n}=3)$ & $27.5 \pm 6.24$ & $45.1 \pm 7.9$ \\
B $(\mathrm{n}=9)$ & $47.7 \pm 2.81$ & $30.2 \pm 4.42$ \\
C $(\mathrm{n}=28)$ & $29.2 \pm 3.3$ & $31.0 \pm 5.3$ \\
\hline
\end{tabular}

cCBF: whole cortex blood flow, tbCBF: thalamusbasal ganglia blood flow.

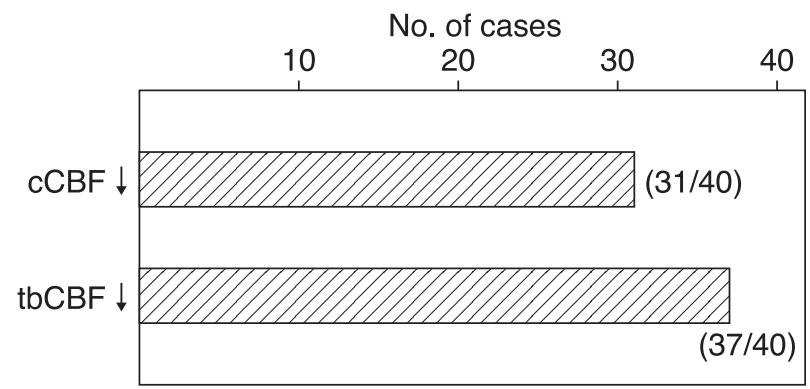

Fig. 5 Preoperative whole cortex blood flow and thalamus-basal ganglia blood flow on $\mathrm{N}$ isopropyl-( $\left.{ }^{123} \mathrm{I}\right)$-P-iodo-amphetamine single photon emission computed tomography by autoradiography method. cCBF $\downarrow$ : group with decrease in whole cortex blood flow, tbCBF $\downarrow$ : group with decrease in thalamusbasal ganglia blood flow.

C: 28 cases) (Fig. 4, Table 2).

The type $C$ cases, the patients with reduced CBF in both the cortex and thalamus-basal ganglia region, were by far the most numerous. Among the three groups combined, the cortical blood flow decreased in 31 patients and the blood flow of the thalamus-basal ganglia region decreased in 37 (Fig. 5).

The assessment of perfusion patterns of the cerebral cortex by 3D-SSP Z-score images revealed a clear cortical hypoperfusion in 31 cases. Three hypoperfusion patterns were noted: the frontal lobe type (F: 19 cases), occipitotemporal lobe type (OT: 5 cases), and mixed type (M: 7 cases). As in our previous report, ${ }^{27)}$ the frontal lobe type was by far the most frequent. At the same time, however, as many as nine cases were assessed as the normoperfusion type $(\mathrm{N})$, exhibiting a pattern with no detectable hypoperfusion in the cortex (Fig. 6). The mean tbCBF was $41.5 \pm 3.52 \mathrm{ml} / 100 \mathrm{~g} / \mathrm{min}$ in the 31 cases with cortical hypoperfusion and $30.5 \pm 4.01 \mathrm{ml} / 100$

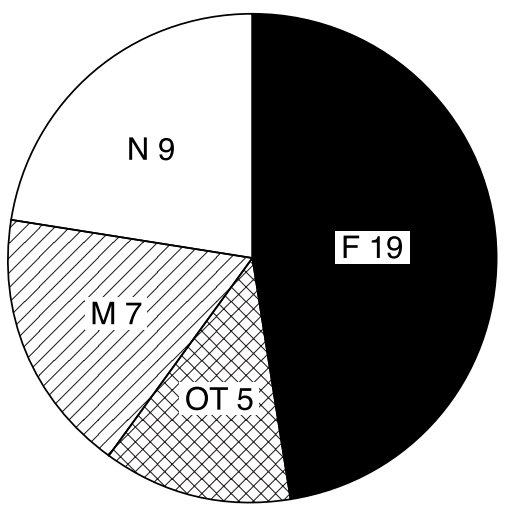

Fig. 6 Preoperative cortical perfusion patterns on $\mathrm{N}$-isopropyl-( $\left.{ }^{123} \mathrm{I}\right)$-P-iodo-amphetamine single photon emission computed tomography three-dimensional stereotactic surface projection Z-score images. F: frontal type, $M$ : mixed type, $N$ : normoperfusion type, OT: occipitotemporal type.

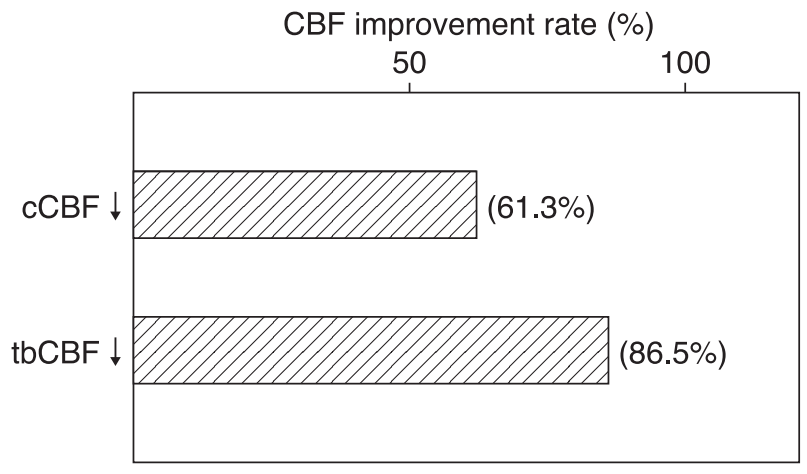

Fig. 7 Postoperative blood flow improvement of whole cortex and thalamus-basal ganglia on $\mathrm{N}$-isopropyl-( $\left.{ }^{123} \mathrm{I}\right)$-P-iodo-amphetamine single photon emission computed tomography by autoradiography method. cCBF $\downarrow$ : group with decrease of whole cortex blood flow, tbCBF $\downarrow$ : group with decrease of thalamusbasal ganglia blood flow.

$\mathrm{g} / \mathrm{min}$ in the nine cases with normoperfusion. The blood flow in the thalamus-basal ganglia region was significantly reduced in the $\mathrm{N}$ type $(\mathrm{p}<0.005)$.

III. Variations of cCBF, tbCBF, and perfusion patterns of the cerebral cortex after surgery

The blood flow patterns of preoperative CBF reduction improved in one of three type $\mathrm{A}$ cases, seven of nine type B cases, and 15 of 28 type $C$ cases. All of the cases without detectable improvement exhibited increased blood flow in non-reduction 


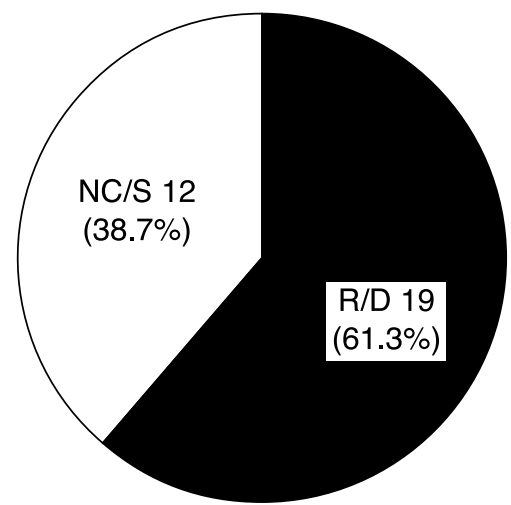

Fig. 8 Postoperative variations of cortical hypoperfusion patterns on $\mathrm{N}$-isopropyl( ${ }^{123}$ I)-P-iodo-amphetamine single photon emission computed tomography threedimensional stereotactic surface projection Z-score images. R/D: reduction or disappearance of hypoperfusion, NC/S: nochange or shift of hypoperfusion site.

areas. The total improvement rates were 19/31 $(61.3 \%)$ in the reduced-cCBF cases and $32 / 37$ (86.5\%) in reduced-tbCBF cases. The blood flow improvement was marked in cases with preoperatively reduced blood flow of the thalamus-basal ganglia region (Fig. 7).

The assessments of the hypoperfusion patterns of the cerebral cortex by 3D-SSP Z-score images in the 31 cases presenting with preoperative cortical hypoperfusion revealed a reduction or disappearance of hypoperfusion in 19 of $31(61.3 \%)$ cases and no-change or a shift of the hypoperfusion site in 12 of 31 (38.7\%) (Fig. 8). The mean tbCBF in the 12 cases of no-change or shift rose from $34.2 \pm 4.63$ $\mathrm{ml} / 100 \mathrm{~g} / \mathrm{min}$ before surgery to $46.7 \pm 3.78 \mathrm{ml} / 100$ $\mathrm{g} / \mathrm{min}$ after surgery. The blood flow in the thalamusbasal ganglia region improved significantly after surgery $(\mathrm{p}<0.003)$ in both the no-change and shift cases.

The reductions of cortical blood flow on ARG method and hypoperfusion of the cerebral cortex on 3D-SSP Z-score images had very favorably correlated patterns both before and after surgery in this study.

\section{Discussion}

In a paper from 2005 we reported two notable results from evaluations of the shunt effect on CBF in shunt-effective iNPH cases: first, that the mean $\mathrm{CBF}$ increases by at least $10 \%$ after the cerebrospinal fluid tap test and increases significantly after surgery; second, that the hypoperfusion pattern of the cerebral cortex by 3D-SSP Z-score images is not localized to the parietal lobe. ${ }^{27)}$ In other respects, however, the results were discrepant: while the symptoms were improved and the mean CBF increased after surgery, the 3D-SSP Z-score images revealed no improvement in the postoperative hypoperfusion pattern of CBF in $40 \%$ of the cases. ${ }^{27)}$ We speculate that the hypoperfusion patterns of the cerebral cortex by 3D-SSP Z-score mainly reflect the cerebral cortical blood flow, while the blood flows in the other regions might increase. We carried out the present study to investigate pathophysiological conditions of cerebral circulatory disorders in iNPH.

The results of our investigation reconfirmed the significant increase of the postoperative mean CBF in the shunt-effective cases. Further, a comparison between the perfusion pattern of the cerebral cortex (assessed by 3D-SSP Z-score images ${ }^{23)}$ ) and the local $\mathrm{CBF}$ in the whole cortical region and thalamus-basal ganglia region (measured by the ARG method $^{8,10}$ ) before surgery revealed two distinct groups: one presenting a reduced blood flow localized in either the cortical region or the thalamus-basal ganglia region, and one with reduced blood flow in both regions. The normoperfusion type $(\mathrm{N})$ was not reflected in the 3D-SSP Z-score images in the group with reduced blood flow localized in the thalamusbasal ganglia region, hence the $\mathrm{N}$ type was only presumed in the 3D-SSP Z-score images of these cases. The post-surgical improvement of CBF appeared in several patterns: in addition to the group with complete CBF improvement in both the whole cortical region and thalamus-basal ganglia region, there were also groups with improvement in only one or the other. Moreover, the significant postsurgical increase of the blood flow in the 3D-SSP Zscore images of the thalamus-basal ganglia regions of the no-change or shift cases indicated a compensatory increase of the blood flow at the preoperative normoperfusion sites.

The cerebral circulatory disorders in iNPH are difficult to explain one-dimensionally from the results of this study. We speculate that there are two pathophysiological conditions: "circulatory disorder in the cerebral cortex" and "circulatory disorder in the thalamus-basal ganglia region." In discussing these two pathologic conditions we must mention the mechanism behind the occurrence of iNPH.

Adams et al. ${ }^{1,2)}$ proposed that iNPH arises as a result of arachnoiditis in the cerebral convexity caused by an unknown etiology. This is refuted, however, by a report ${ }^{33}$ ) arguing that circulatory disorders of the cerebrospinal fluid itself confer no 
effects. ${ }^{6)}$ In any case, we can surmise that some disorder of the absorbance mechanism of cerebrospinal fluid may be present. ${ }^{11,25)}$ Intriguingly, Kondo ${ }^{15)}$ hypothesized that the shift of blood from the bridging vein to the sinus may be induced by the balance among the pressures of the bridging vein, sinus, cerebrospinal fluid in the subarachnoid cavity, and wash-out mechanism of expired and inspired respiration. If this hypothesis is correct, any variation in the pressure of the cerebrospinal fluid in the subarachnoid cavity resulting from the iNPHinduced disorder of cerebrospinal could disturb the pressure balance of the wash-out mechanism. This condition would presumably induce collapse and reduced compliance of the cortical veins, including the bridging vein, ${ }^{3-5)}$ and consequently change the disorder from a hypoperfusion of the cortical vein to a disorder of the cortical circulation. This is assumed to be the pathophysiological condition of the "circulatory disorder in the cerebral cortex." On the other hand, the expansion of the lateral ventricle and third ventricle induced by the circulatory disorder of the cerebrospinal fluid leads to a pressure of the peripheral cerebral tissues, which thus changes the disorder to a circulatory disorder of the tissues around the ventricle due to the hypoperfusion caused by the compression and ensuing lesser pathway. This is assumed to be the pathophysiological condition of the "circulatory disorder in the thalamus-basal ganglia region." The interstitial fluid bulk flow ${ }^{3)}$ accompanying the circulatory disorder in the cerebral cortex and the lesser pathway from the ventricle eventually induces the circulatory disorder of the white matter and the paraventricular watershed region, ${ }^{7,24,32)}$ leading to a circulatory disorder of the whole brain (Fig. 9).

Earlier studies on ischemic sites in iNPH before surgery $^{16,17,19,20,22,24,26-32,34)}$ have reported various patterns of these two pathophysiological conditions according to the disease stage of iNPH. While one group reports only reductions in hypoperfusion, ${ }^{34)}$ most authors agree that the CBF improves after surgery in shunt-effective cases, in light of the compensatory increase of the blood flow at normoperfusion sites in non-improved hypoperfusion cases after surgery. ${ }^{9,12,13,16-18,20,21,28-32,34)}$

The mCBF, $\mathrm{cCBF}$, and tbCBF were measured by the ARG method before and after surgery in 40 patients with possible iNPH who responded well to shunt treatment. And the results were compared with the perfusion patterns of the cerebral cortex by 3D-SSP Z-score images. The following conclusions were obtained: 1) In shunt effective cases, the postoperative mCBF increased in shunt-effective cases. 2) The pattern of blood flow reduction in the

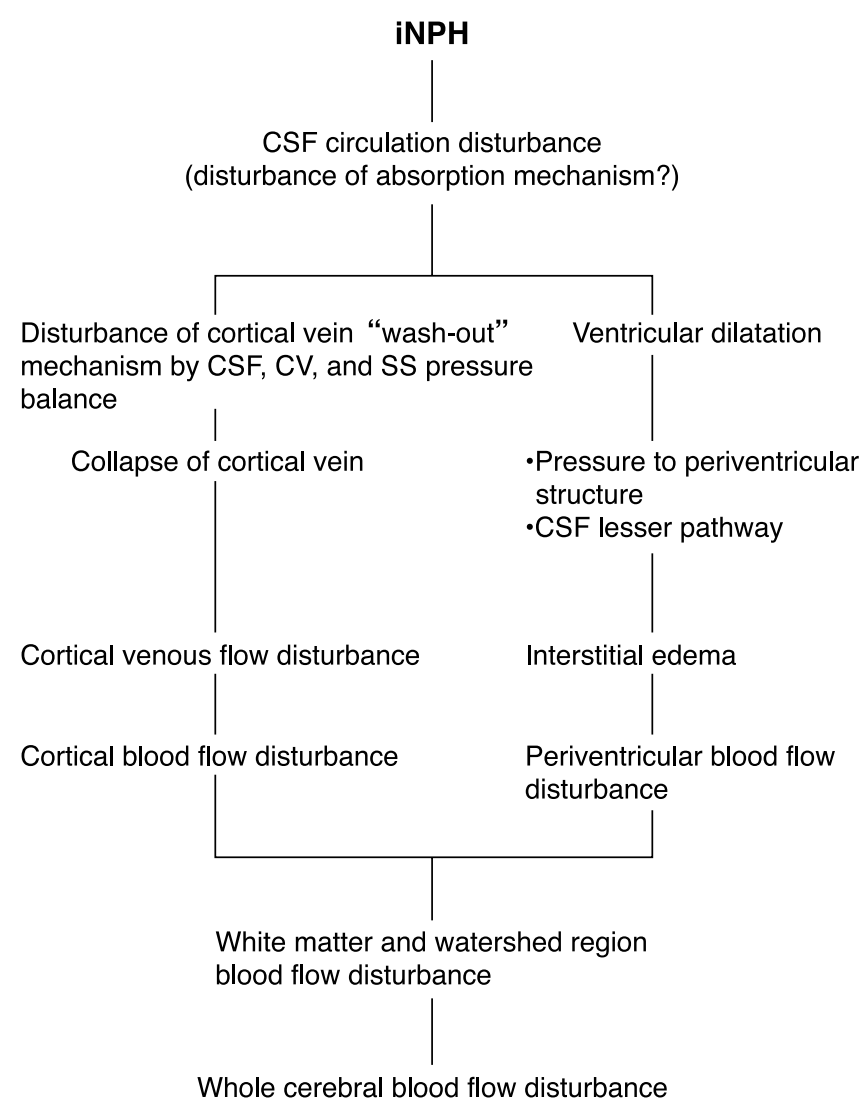

Fig. 9 Mechanisms of cerebral blood flow disturbance in idiopathic normal pressure hydrocephalus (iNPH). CSF: cerebrospinal fluid, CV: cortical vein, SS: sagittal sinus.

cerebral cortex assessed by the ARG method correlated with the pattern of hypoperfusion of the cerebral cortex assessed by 3D-SSP Z-score images. 3) The blood flow was significantly reduced in the thalamus-basal ganglia of the cases exhibiting normoperfusion of the cerebral cortex on 3D-SSP Z-score images. 4) The CBF improved postoperatively in the thalamus-basal ganglia region, in the cerebral cortex region, or in both regions. 5) A compensatory increase of blood flow was found in other normoperfusion sites in cases without postoperative improvement of the hypoperfusion sites. 6) The blood flow in the thalamus-basal ganglia region increased significantly after surgery in all the cases manifesting either no-change or a shift of hypoperfusion sites on 3D-SSP Z-score images. 7) Cerebral circulatory disorders in iNPH manifest as either of two pathophysiological conditions: the "circulatory disorder of the cerebral cortical region" and the "circulatory disorder of the thalamus-basal ganglia region." Various patterns develop according to the disease stage. 


\section{Acknowledgments}

Part of this study was carried out under a project to create a unified standard for the diagnosis and treatment of iNPH by the Japan Society of Normal Pressure Hydrocephalus (study of idiopathic normal pressure hydrocephalus on neurological improvement: SINPHONI).

\section{References}

1) Adams RD: Recent observations on normal pressure hydrocephalus. Schweiz Arch Neurol Neurochir Psychiatr 116: 7-15, 1975

2) Adams RD, Fisher CM, Hakim S, Ojemann RG, Sweet WH: Symptomatic occult hydrocephalus with "normal" cerebrospinal-fluid pressure: a treatable syndrome. N Engl J Med 273: 117-122, 1965

3) Bateman GA: Vascular compliance in normal pressure hydrocephalus. AJNR Am J Neuroradiol 21: 1574-1585, 2000

4) Bateman GA: Pulse-wave encephalopathy: a comparative study of the hydrodynamics of leukoaraiosis and normal-pressure hydrocephalus. Neuroradiology 44: 740-748, 2002

5) Bateman GA: The reversibility of reduced cortical vein compliance in normal-pressure hydrocephalus following shunt insertion. Neuroradiology 45: 65-70, 2003

6) Bech RA, Juhler M, Waldemar G, Klinken L, Gjerris F: Frontal brain and leptomeningeal biopsy specimens correlated with cerebrospinal fluid outflow resistance on normal pressure hydrocephalus. Neurosurgery 40: 497-502, 1997

7) Guideline Committee for Idiopathic Normal Pressure Hydrocephalus: [Clinical Guidelines for Idiopathic Normal Pressure Hydrocephalus]. Osaka, Medical Review, 2004 (Jpn)

8) Hatazawa J, Iida $H$, Shimosegawa E, Sato $T$, Murakami M, Miura Y: Regional cerebral blood flow measurement with iodine-123-IMP autoradiography: normal values, reproducibility and sensitivity to hypoperfusion. J Nucl Med 38: 1102-1108, 1997

9) Hertel F, Walter C, Schmitt M, Morsdorf M, Jammers W, Busch HP, Bettag M: Is a combination of TcSPECT or perfusion weighted magnetic resonance imaging with spinal tap test helpful in the diagnosis of normal pressure hydrocephalus? J Neurol Neurosurg Psychiatry 74: 479-484, 2003

10) Iida $\mathrm{H}$, Itoh $\mathrm{H}$, Nakazawa $M$, Hatazawa J, Nishimura $\mathrm{H}$, Onishi Y, Uemura K: Quantitative mapping of regional cerebral blood flow using iodine-123-IMP and SPECT. J Nucl Med 35: 2019-2030, 1994

11) Kitagami H, Mori E: CSF spaces in idiopathic normal pressure hydrocephalus: morphology and volumetry. AJNR Am J Neuroradiol 19: 1277-1283, 1998

12) Klinge $P$, Berding $G$, Brinker $T$, Schuhmann $M$, Knapp WH, Samii M: PET-studies in idiopathic chronic hydrocephalus before and after shunt-treat- ment: the role of risk factors for cerebrovascular disease (CVD) on cerebral hemodynamics. Acta Neurochir Suppl 81: 43-45, 2002

13) Klinge P, Berding G, Brinker T, Weckesser E, Knapp WH, Samii M: Regional cerebral blood flow profiles of shunt-responder in idiopathic chronic hydrocephalus - a 15-O-water PET-study. Acta Neurochir Suppl 81: 47-49, 2002

14) Klinge P, Ruckert N, Schuhmann M, Berding G, Brinker T, Knapp WH, Samii M: Neuropsychological sequels to changes in global cerebral blood flow and cerebro-vascular reserve capacity after shunt treatment in chronic hydrocephalus - a quantitative PET-study. Acta Neurochir Suppl 81: 55-57, 2002

15) Kondo T: [Mechanisms of cerebral venous system on cerebral circulation]. Nichidai Igaku Zasshi 37: 1-21, 1978 (Jpn, with Eng abstract)

16) Kristensen B, Malm J, Fagerland M: Regional cerebral blood flow, white matter abnormalities, and cerebrospinal fluid hydrodynamics in patients with idiopathic adult hydrocephalus syndrome. J Neurol Neurosurg Psychiatry 60: 282-288, 1996

17) Larsson A, Bergh AC, Bilting M: Regional cerebral blood flow in normal pressure hydrocephalus: diagnostic and prognosis aspects. Eur J Nucl Med 21: 118-123, 1994

18) Mamo HL, Meric PC, Ponsin JC: Cerebral blood flow in normal pressure hydrocephalus. Stroke 18: 1074-1080, 1987

19) Mataro M, Poca MA, Salgado-Pineda P, CastellConesa J, Sahuquillo J, Diez-Castro MJ, Aguade-Bruix S, Vendrel P, del Mar Matarin M, Junque C: Postsurgical cerebral perfusion changes in idiopathic normal pressure hydrocephalus: a statistical parametric mapping study of SPECT images. J Nucl Med 44: 1884-1889, 2003

20) Meyer JS, Kitagawa Y, Tanahashi N: Evaluation of treatment of normal-pressure hydrocephalus. $J$ Neurosurg 62: 513-521, 1985

21) Meyer JS, Kitagawa Y, Tanahashi N: Pathogenesis of normal-pressure hydrocephalus: preliminary observation. Surg Neurol 23: 121-133, 1985

22) Meyer JS, Tachibana H, Hardenbarg JP: Normal pressure hydrocephalus: Influences on cerebral hemodynamic and cerebrospinal fluid pressure: chemical autoregulation. Surg Neurol 21: 195-203, 1984

23) Minoshima S, Frey KA, Koeppe RA, Foster NL, Kuhl DE: A diagnostic approach in Alzheimer's disease using three-dimensional stereotactic surface projection of fluorine-18-FDG PET. J Nucl Med 36: 1238-1248, 1995

24) Momjian S, Owler BK, Czosnyka Z, Czosnyka M, Pena A, Pickard JD: Pattern of white matter regional cerebral blood flow and autoregulation on normal pressure hydrocephalus. Brain 127: 947-948, 2004

25) Mori E: [Mechanisms of idiopathic normal pressure hydrocephalus]. Naika 95: 803-808, 2005 (Jpn)

26) Owler BK, Momjian S, Czosnyka Z, Czosnyka M, Pena A, Harris NG, Smielelwski P, Fryer T, Donovan T, Coles J, Carpenter A, Pickard JD: Normal pressure 
hydrocephalus and cerebral blood flow: a PET study of baseline values. J Cereb Blood Flow Metab 24: 17-23, 2004

27) Takeuchi T, Ishihara T, Kudo H, Izawa N, Watanabe C, Tanno Y, Abe Y, Katayama S, Goto H, Izaki K, Kokubu K, Oda M, Ohmae T, Sasanuma J, Maeno K, Kikuchi Y, Koizumi J, Watanabe Z, Ito Y, Ohara H, Kowada M, Watanabe K: [Significance of shunt efficacy decision of SPECT on idiopathic normal pressure hydrocephalus]. No To Shinkei 57: 306-312, 2005 (Jpn, with Eng abstract)

28) Takeuchi T, Kasahara E, Iwasaki M: [Clinical characteristics and indications for shunting in patients with idiopathic normal pressure hydrocephalus with brain atrophy (atypical idiopathic normal pressure hydrocephalus)]. No Shinkei Geka 28: 505-515, 2000 (Jpn, with Eng abstract)

29) Takeuchi T, Kasahara E, Iwasaki M, Mima T, Mori $\mathrm{K}$ : Indications for shunting in patients with idiopathic normal pressure hydrocephalus presenting with dementia and brain atrophy (atypical idiopathic normal pressure hydrocephalus). Neurol Med Chir (Tokyo) 40: 38-47, 2000

30) Takeuchi T, Kasahara E, Takahashi E, Kojima S, Ogawa H, Kuzuki K, Miyamae T, Yamazaki S: [Semiquantifying regional cerebral blood flow by dynamic CT scanning: correlation with ${ }^{123}$ I-amphetamine (IMP) SPECT]. CT Kenkyu 12: 555-561, 1990 (Jpn, with Eng abstract)

31) Tamaki N, Kusunoki T, Wakabayashi T: Cerebral hemodynamics in normal-pressure hydrocephalus: evaluation by ${ }^{133} \mathrm{Xe}$ inhalation method and dynamic CT study. J Neurosurg 61: 510-514, 1984

32) Tanaka A, Kimura M, Nakayama Y, Yoshinaga S, Tomonaga M: Cerebral blood flow and autoregulation in normal pressure hydrocephalus. Neurosurgery 40: 1161-1167, 1997

33) Vanneste JA: Diagnosis and management of normalpressure hydrocephalus. J Neurol 247: 5-11, 2002

34) Vorstrup S, Christensen J, Gjerris F: Cerebral blood flow in patients with normal-pressure hydrocephalus before and after shunting. J Neurosurg 66: 379-387, 1987

Address reprint requests to: Totaro Takeuchi, M.D., Department of Neurosurgery, Gyoda General Hospital, 376 Mochida, Gyoda, Saitama 361-0056, Japan.

e-mail: t.takeuchi@gyoda-hp.or.jp

\section{Commentary}

This article is an important contribution to the pathophysiology of NPH. We were fortunate enough to work in Boston during the time that Dr. Hakim and Professor Ray Adams described this new syndrome. The authors have elegantly confirmed that iNPH with decreased CBF before surgery undergo postoperative increase in BF when shunts are effective, as has been shown in the extensive literature. They have divided the perfusion patterns into three groups, with discrepant results, reconfirming the significant increase of the postoperative mean CBF in the shunt-effective cases, demonstrating that there are two main pathophysiological conditions: a) circulatory disorders in the cerebral cortex, and b) in the thalamus-basal ganglia region, thus creating a unified standard for the diagnosis and treatment of iNPH.

Raul MARINO, Jr., M.D. Department of Neurosurgery University of São Paulo Medical School Instituto Neurológico de São Paulo São Paulo, Brazil

Takeuchi et al. present interesting results of a study on regional cerebral blood flow (CBF) before and after shunting procedure in 40 patients with idiopathic normal pressure hydrocephalus (iNPH). CBF study using ${ }^{123}$ I-IMP SPECT showed a decrease in CBF in the cerebral cortex and/or thalamus-basal ganglia in all of the patients preoperatively. After surgery, an increase in CBF in $61.3 \%$ of the 31 patients with decreased cortical blood flow and in $86.5 \%$ of the 37 patients with decreased blood flow in the thalamus-basal ganglia was observed. Furthermore, 3D-SSP Z-score images revealed a decrease or disappearance of cortical hypoperfusion area in $61.3 \%$ of 31 patients with preoperative cortical hypoperfusion after surgery. The authors refer to the mechanism of CBF disturbance in patients with iNPH, based on their data that there are three patterns in decreased regional CBF, which are decreases in CBF at the cortex, thalamusbasal ganglia or both.

Idiopathic NPH is a treatable neurological disorder characterized by gait disturbance, incontinence, and dementia. However, although we have recently published elaborate guidelines for diagnosis and management of iNPH, the preoperative diagnosis of iNPH remains controversial without definite criteria. The correlation between clinical symptoms and hemispheric or regional CBF in iNPH has not been established. This article demonstrates that decreased regional CBF is not the consequence of neural dysfunction but is associated with a causative mechanism of clinical symptoms in iNPH, because regional CBF improved after surgery in most of the patients who were judged to show shunt effectiveness. The authors have made a valuable contribution to better understanding of the pathophysiology of iNPH.

Kikuo OHNO, M.D. Neurosurgery

Department of Brain Medical Science Division of Cognitive and Behavioral Medicine Graduate School Tokyo Medical and Dental University Tokyo, Japan 